\title{
Riboflavin and Ultraviolet A radiation cross- linking for keratoconus management: a review*
}

\author{
AME van $\mathrm{Zyl}^{\mathrm{a}}$ and WDH Gillan ${ }^{\mathrm{b}}$ \\ Department of Optometry, University of Johannesburg, PO Box 524, Auckland Park, 2006 South \\ Africa \\ <wgillan@uj.ac.za>
}

Received 17 January 2011; revised version accepted 31 October 2012

\section{Introduction}

Keratoconus is a non-inflammatory ectasia that results in distorted and decreased vision due to progressive thinning, protrusion and scarring of the cornea ${ }^{1}$. Keratoconus is not predisposed to race or gender and is found in approximately one in 2000 members of the general population ${ }^{2}$. The cause and pathogenesis of keratoconus is still unclear, however, aspects like eye rubbing, decreased ocular rigidity, abnormalities of connective tissue, the role of degradative enzymes and protein inhibitors, genetics as well as the possible role of interleukin 1 are considered important ${ }^{1,2}$. Keratoconus is commonly seen as an isolated disorder yet has been found in conjunction with mitral valve prolapse ${ }^{3}$, Down's syndrome, Leber's congenital amaurosis, atopic disease and connective tissue diseases ${ }^{1,2}$. The treatment options for keratoconus depend on the stage of the disease. Initially spectacles and soft contact lenses may be used to improve vision. As the irregular astigmatism increases, however, vision will no longer be good enough and hard contact lenses, scleral lenses or hybrid lenses will be the next options. With further progression the cornea becomes too thin or scarred resulting in the need for corneal transplant as a last resort (this could be penetrating or lamellar depending on the damage to the cornea). The progression of keratoconus normally stops in the third or fourth decade of life and is due to corneal ageing where natural cross-linking causes spontaneous biomechanical and biochemical stability of the disease $2,4,5$. In diabetic patients keratoconus is very rarely found because of possible advanced glycation end products causing natural cross-linking, which increases corneal rigidity 5,6 . The effects of ageing and diabetes on the cornea suggest that riboflavin ultraviolet cross-linking (CXL) may be the best way to stop the progression of keratoconus, remembering however that keratoconus is not yet curable ${ }^{7}$. The aim of CXL is to alter the stromal composition by inducing cross-links between the collagen fibrils that may increase the tensile strength of the cornea, and stop thinning and thus the progression of keratoconus ${ }^{7,8}$. In the 1990 's the technique for corneal collagen cross-linking with Riboflavin and UVA radiation was developed in Germany at the Dresden Technical University and in 1998 the first patient with keratoconus was treated with the procedure ${ }^{7,9}$.

\section{Procedure of CXL}

The procedure is done on an outpatient basis. Topical anesthesia is administered to the eye and thereafter epithelial tissue is mechanically removed with a blunt instrument in a 7-9 $\mathrm{mm}^{6,7,9-18}$ diameter zone (the epithelium can be debrided with a variety of tools: a Beaver blade $^{6}$, Amoil brush ${ }^{9}, 10$, rotating soft brush ${ }^{13}$, blunt spatula $11,14,15,18,19$, blunt hockey knife ${ }^{20}$, a blunt crescent knife ${ }^{21}$ or a blunt knife ${ }^{12,16}$ ). The epithelium is a diffusion barrier to the cornea which needs to be removed to aid the penetration of riboflavin, which is water soluble 22 , into the cornea. Riboflavin solution is applied before and during irradiation every 3-5 
minutes $6,13,15,19,23$, starting 5-40 minutes before irradiation $6,7,11-13,19,23$. The eye is examined under a slitlamp using blue light to look for yellow colouring of the aqueous which ensures full penetration of riboflavin into the anterior chamber. The lids are then separated with a lid speculum. Irradiation is performed $6,7,9-15,18,19,23$ at a distance of $1-5 \mathrm{~cm}^{18,19,23}$ from the eye for 30 minutes using a UVA double diode at $370 \mathrm{~nm}$ and an irradiance of $3 \mathrm{~mW} / \mathrm{cm}^{2}$. A bandage contact lens is usually placed on the cornea post-operatively ${ }^{9,16}$, mostly for three to five days $6,11,14,18,23$, or until reepithelialization is complete ${ }^{10,13,15}$. Reepithelialization can take anything from one to four days ${ }^{4,10,12}$. No persistent epithelial deficit has been found post-operatively $12,13,16,18$.

Alternative protocols have been used. In three studies ${ }^{9-11}$ pilocarpine drops were instilled to reduce the amount of UVA light entering the eye and protect the ocular lens. Caporossi et al ${ }^{14}$ used a procedure that was unique. They interrupted application of radiation every five minutes by turning the diodes off, instilling riboflavin in the eye, and then continuing. What is important is to ensure full penetration of the cornea with riboflavin. The UVA source must be used with a potentiometric voltage regulator and the device must be properly calibrated with a UVA power meter prior to every patient being radiated. This will ensure that every patient receives 5,15 a uniform homogeneous level of UVA radiation of $370 \mathrm{~nm}$ with irradiance of $3 \mathrm{~mW} / \mathrm{cm}^{2}$.

Generally, UV radiation represents a danger to the eye but this is mostly true for UVB radiation (290$320 \mathrm{~nm}) .{ }^{12}$ UVA radiation $(320-400 \mathrm{~nm})$ alone has a damage threshold value of $4 \mathrm{~mW} / \mathrm{cm}^{2}$ at the cornea ${ }^{16}$. When riboflavin is instilled into the cornea the damage threshold 2,12 drops to $0.35 \mathrm{~mW} / \mathrm{cm}$. During cross-linking UVA radiation at $370 \mathrm{~nm}$ is used as this wavelength is optimally absorbed by riboflavin thus ensuring that approximately $95 \%$ of the UV radiation is absorbed in the cornea ${ }^{7,19}$.

Riboflavin acts as a photo-sensitizer when combined with UVA light ${ }^{7}, 10$. Riboflavin is excited into its triplet state generating reactive oxygen species (ROS), comprised of singlet oxygen and a few superoxide anion radicals. These ROS react with molecules in the cornea causing increased covalent bonds to form between amino groups of collagen fibrils ${ }^{5}$. These covalent bonds then change tissue properties and stabilize the collagen scaffold. This results in an increase in rigidity of the cornea, increased resistance against proteolytic enzymes ${ }^{7}$ and a significant increase in the diameter of collagen fibers ${ }^{5}$.

\section{Safety concerns on UVA radiation}

The cross-linking effect only occurs in the anterior $200-300 \mu \mathrm{m}$ of the cornea ${ }^{8,11,20}$ which is due to the high levels of absorption of UVA radiation in this area $^{24}$. This ensures that more posterior structures (for example, the endothelium, lens and retina) are not adversely affected, however, an absolute minimal corneal thickness of $400 \mu \mathrm{m}$ is required to diminish potential for damage $3,5,7,12,19$. If this value is not respected the treatment may induce cataracts or even retinal damage. Damage could also arise when a patient does not maintain fixation on the focus point resulting in the limbus being irradiated. In these low-compliance patients a poly-methyl methacrylate ring can be placed over the limbus to ensure limbal protection. Under normal conditions (adequate fixation) the limbus is not exposed to the effects of UVA radiation. Less than $20 \mu \mathrm{m}$ lateral diffusion (from the edge of the abraded area) of radiation effects occur. The protection is provided by the presence of the epithelium ${ }^{11}$.

Wollensak et $a l^{20}$ measured the cytotoxicity of different irradiance levels of UVA, different concentrations of riboflavin and the combination of one concentration of riboflavin and different UVA irradiance levels. No cytotoxicity was found for riboflavin alone. The cytotoxicity level for UVA in combination with riboflavin was found to be 10 times lower than with UVA alone. This level of cytotoxic effect did happen gradually but at a distinct threshold of irradiance level. The cytotoxicity is caused by the oxidant effect of UVA radiation. In human corneas the cytotoxic effect on keratocytes reaches approximately $300 \mu \mathrm{m}$ deep into the cornea when the proposed protocol is used ${ }^{21,20}$. When using the protocol specified and threshold minimal thickness is adhered to, the treatment is seen as safe $5,6,8,11,18,19$ and safer than corneal transplant ${ }^{6}$.

\section{Keratocytes}

In a normal cornea the distribution of keratocytes 
is denser in the anterior cornea decreasing in density towards the posterior cornea ${ }^{13}$. Removing the epithelial layer results in keratocyte apoptosis (the process of programmed cell death) in the anterior $50 \mu \mathrm{m}$ of the cornea ${ }^{18,21}$. One month post-operatively keratocyte apoptosis was noted to a corneal depth of approximately $300-350 \mu \mathrm{m}^{13}, 15,18$. Repopulation of keratocytes was found to occur in the anterior cornea and mid-stroma in the following few months and was associated with corneal oedema ${ }^{8,10,13,18}$. It has been shown that the repopulation of keratocytes is initiated from activated keratocytes in deeper layers of the stroma $^{18}$. Stroma deeper than approximately $350 \mu \mathrm{m}$ showed an increased density of keratocytes in the first and third post-operative months ${ }^{13}$. Stromal repopulation was almost complete within six months ${ }^{15,18}$.

Stromal density is increased after CXL is performed because well-structured compact collagen lamellae are produced after repopulation of keratocytes, which is seen as haze ${ }^{11}$. Even though cross-linking does induce cellular apoptosis, the regeneration processes of these cells result in normal tissue histology ${ }^{8}$.

\section{Stromal thickness}

Numerous studies have reported on a decrease in corneal thickness one year after $\mathrm{CXL}^{9}, 10,16,17$ while others $^{6,19,21}$ have found that corneal thickness does not change post-operatively. Optical Coherence Tomography (OCT) has been used to show a significant increase in corneal thickness six months post-operatively. It is believed that this increase is caused by keratocytes producing new proteoglycans. The increase can be seen as an indication that the decrease in corneal thickness after one year is not an indication of progression of keratoconus, as the normal progression of keratoconus is not linked with a period of increased thickness but only thinning ${ }^{16}$. Alternatively the early increase of stromal thickness could be a measurement artifact seen in most patients post-operatively because of haze. This artifact is not present one year post-operatively, because of the disappearance of haze, seen with slit-lamp observation ${ }^{17}$.

\section{Corneal transparency and endothelium}

In two studies ${ }^{10,11} 11-12.6 \%$ of the eyes treated developed corneal haze which was successfully managed with a regimen of topical steroids. El-Ragga ${ }^{16}$ observed faint diffuse haze post-operatively, in all his subjects, that spontaneously disappeared within one month. One eye, however, was left with a faint superficial corneal scar. The post-operative haze seems to be more prevalent in subjects with more advanced keratoconus ${ }^{18}$. In other studies ${ }^{11,25}$ hyperactivated keratocyte nuclei in the anterior stroma (to a depth of $80 \mu \mathrm{m}$ ), and reticular patterned dark microstriae (with or without Vogt's striae) were identified as possible risk factors for corneal opacity when observed preoperatively. The haze seemed to not decrease these patients vision. The risk factors are important as these patients are more likely to not only develop haze but late stromal scarring post-operatively as well.

Post-operatively few clinical changes ${ }^{17,18,25}$ morphological changes ${ }^{13,14}$ or changes in cell count have been noted in the endothelium following $\mathrm{CXL}^{9}, 10,12,14$. In one study ${ }^{16}$, one out of 29 eyes treated presented with endothelial irregularities one month post-operatively. These irregularities disappeared two months later without any visual hindrance. The most important factor in reducing radiation and thus cytotoxic effects on the endothelium is to ensure a minimal inclusion thickness of $400 \mu \mathrm{m}$ for all CXL patients?

\section{Regression of keratometric (K)-readings}

Progression of keratoconus can be established by the ongoing steepening of the K-values of the cornea. After CXL it has been found that K-readings flattened $^{9,10,17}$ or stabilized ${ }^{6,7}$ and that the cornea had a tendency to take on a more symmetric form ${ }^{6,13,14}$. In a few rare cases a steepening of K-readings was found. Wollensak et al ${ }^{12}$ observed a minimal increase in one out of 23 eyes examined. It is important to realize that a flattening effect of a keratoconic cornea has never before been documented and that control groups of keratoconic eyes showed definite progression. This flattening has been called post-operative regression which was found in $70 \%$ of CXL patients ${ }^{12}$.

\section{Stromal demarcation line}

Changes in the corneal microstructure do not occur throughout the entire cornea. The transition between the treated zone and untreated zone of the cornea is rapid $^{18,22}$ and is normally less than $350 \mu \mathrm{m}$ into the cornea. Initially the anterior stroma shows oedema and hypo-reflectivity while the posterior stroma is 
normal. The transition can be seen as an opaque/ whitish line when using an OCT for instance, because of the rapid change between the two zones. Generally, the demarcation line disappears within three months post-operatively ${ }^{16,22}$. The average depth of this line is approximately $313 \mu \mathrm{m}^{16,17}$. From the third to sixth months post-operatively the reflectivity of the anterior area changes from hypo- to hyper-reflectivity because of increased density in this part of the cornea. The line between the normal density cornea and the hyper reflective area is now called the late demarcation line. This late demarcation line can be detected for up to three years post-operatively when using confocal laser scanning microscopy ${ }^{11}$.

\section{Subepithelial plexus}

One month post-operatively there is an absence of the subepithelial nerve plexus in the treatment area when observed with a confocal microscope ${ }^{11,13}$. Regeneration starts within the next few months. The nerves grow from the surrounding area that had not been irradiated. The anterior midstromal nerve fibers are also absent post-operatively but these are regenerated from the deep stromal nerve plexus ${ }^{13}$. After six months the cornea's sensitivity is restored to normal and reinnervation is nearly complete. Only two years after CXL does the nerve plexus fully resemble the pre-operative structure ${ }^{11}$.

\section{Intra Ocular Pressure}

Several studies have shown that no statistically significant changes take place in intra ocular pressure (IOP) measurements following $\mathrm{CXL}^{7}, 12,14,16,19$. Goldich et $a^{23}$, however, showed an increase in post-operative IOP using contact and noncontact IOP measurement techniques. It could not be concluded, however, whether it was a true elevation in IOP or an increase due to the stiffening effect caused by CXL.

\section{Visual Acuity}

Uncorrected visual acuity (UCVA) was statistically significantly improved by approximately 1-1.3 lines $6,10,14$, best corrected visual acuity (BCVA) had a statistically significant increase of approximately 1.26 lines $^{8,12}$ and best corrected spherical visual acuity (BCSVA) remained stable ${ }^{14,16}$ when comparing pre- and post-operative values.

Interestingly, Doors et a ${ }^{16}$ found a significant decrease in BCSVA one month post-operatively but at three, six and 12 months post-operatively the BSCVA was stable compared to pre-operative values. The decrease at one month was attributed to the remodeling process taking place in the cornea. It seems that the improvement in VA happens in the first three to 12 months post-operatively and then remains unchanged ${ }^{7}, 10$. The methods used to measure these changes varied, in one study Snellen charts were used ${ }^{14}$ in others $\log$ MAR charts ${ }^{6,7,10}$ and one study measured visual acuity with a Snellen chart, but converted the data into $\log$ MAR for comparison of data 22 .

\section{Refractive status after procedure}

A slow but significant improvement in spherical refractive error $6,9,12,14,16,19$ and cylindrical refractive error $9,10,14$ was seen in as little as three months postoperatively when compared to mean pre-operative values. However two studies 9,10 found an axis shift post-operatively. The methods of determining the refractive change varied from subtracting data collected at follow-up examinations from data collected on the day of the procedure ${ }^{7}$ to converting the data into vectors and then calculating the change found on followup visits when comparing data to the data collected on the day of the procedure ${ }^{16}$.

\section{Complications of CXL}

The most common complication evaluated was haze $6,10,11,18,25$ and some patients complained of haloes and night glare for the first three months postoperatively ${ }^{10}$. Additional complications were postoperative herpetic keratitis with iritis in a patient with no history of herpetic disease ${ }^{26}$ and diffuse lamellar keratitis in a patient treated with CXL for post-laser in situ keratomileusis ${ }^{27}$.

\section{Conclusion}

CXL seems to be the only form of treatment available to stop the progression of keratoconus. As the progression of keratoconus leads to a decrease of vi- 
sion and inability of vision to be adequately restored with spectacles and soft contact lenses, it is important to do the procedure as soon as keratoconus is diagnosed $^{12,16}$ and before the cornea thins too much. Computer-assisted videokeratoscopes are the most sophisticated and sensitive devices for diagnosing irregular astigmatism which is the hallmark sign of keratoconus $^{2}$. Early CXL treatment will preserve the patient's vision at a better level12, 16. This procedure should only be done if progressive keratoconus has been established, and if the cornea is not thinner than $400 \mu \mathrm{m}^{5}, 6,8,11,14$. Most keratoconic patients over the age of 35 years no longer have progression of keratoconus thus patient selection is very important.

There is a debate between ophthalmologists whether to remove the epithelium or not when doing the procedure because of the pain that patients have for approximately a day post-operatively. It has been concluded that removal of the epithelium is necessary for the absorption of riboflavin by the stroma ${ }^{11}$.

The main aim of the procedure initially was to stabilize the keratoconic cornea. Stabilization was achieved with extra benefits like more symmetric corneas, which not only increased VA but made the cornea easier to fit with contact lenses. It is reported that the cornea still tolerates contact lenses after the procedure $^{12}$. Some investigations indicate that keratoconus leads to keratoplasty in approximately $20 \%$ of patients ${ }^{2}, 5,10,22$. CXL will significantly decrease the need for keratoplasty 1, 7, 18, 19,23,24 or at least delay the need for it, which is especially important in third world countries where donor corneas are not easy to come by. CXL is still a new procedure and longer follow-up studies with bigger population groups are necessary, especially because the durability of the cross-linking effect is unknown and a repeated procedure may be necessary.

CXL is a simple and low cost procedure ${ }^{12}$ with a short recovery time and when looking at the research comparing the positive and negative aspects it seems that it should be the first line of action for any patient with keratoconus.

\section{References}

1. Krachmer JH, Feder RS, Belin MW. Keratoconus and related noninflammatory corneal thinning disorders. Surv Ophthalmol 198428 293-322.

2. Rabinowits YS. Keratoconus. Surv Ophthalmol 199842 297-319.

3. Sharif KW, Casey TA, Coltart J. Prevalence of mitral valve prolapse in keratoconus patients. J R Soc Med 199285 446448.

4. Daxer A, Misof K, Grabner B, Ettl A, Fratzl P. Collagen fibrils in the human corneal stroma: structure and aging. Invest Ophthalmol Vis Sci 199839 644-648.

5. Wollensak G. Crosslinking treatment of progressive keratoconus: new hope. Curr Opin Ophthalmol 200617 356-360.

6. El-Raggal TM. Riboflavin-ultraviolet A corneal cross-linking for keratoconus. Middle East Africa J Ophthalmol 2009 16 256-259.

7. Raiskup-Wolf F, Hoyer A, Spoerl E, Pillunat LE. Collagen crosslinking with riboflavin and ultraviolet-A light in keratoconus: Long-term results. J Cat Ref Surg 200834 796-801.

8. Tomkins O, Garzozi HJ. Collagen cross-linking: strengthening the unstable cornea. Clinical Ophthalmol 20082863 867.

9. Vinciguerra P, Albè E, Trazza S, Seiler T, Epstein D. Intraoperative and postoperative effects of corneal collagen cross-linking on progressive keratoconus. Arch Ophthalmol 2009127 1258-1265.

10. Vinciguerra $P$, Albè E, Trazza S, Rosetta $P$, Vinciguerra R, Seiler T, Epstein D. Refractive, topographic, tomographic, and aberrometric analysis of keratoconic eyes undergoing corneal cross-linking. Ophthalmol 2009116 369-378.

11. Mazzotta C, Traversi C, Baiocchi S, Caporossi O, Bovone C, Sparano MC, Balestrazzi A, Caporossi A. Corneal healing after riboflavin ultraviolet-A collagen cross-linking determined by confocal laser scanning microscopy in vivo: early and late modifications. Am J Ophthalmol 2008146 527-533.

12. Wollensak G, Spoerl E, Seiler T. Riboflavin/ultraviolet-Ainduced collagen crosslinking for the treatment of keratoconus. Am J Ophthalmol 2003135 620-627.

13. Kymionis G, Diakonis VF, Kalyvianaki M, Portaliou D, Siganos C, Kozobolis V, Pallikaris AI. One-year follow-up of corneal confocal microscopy after corneal cross-linking in patients with post laser in situ keratomileusis ectasia and keratoconus. Am J Ophthalmol 2009 147 774-778.

14. Caporossi A, Baiocchi S, Mazzotta C, Traversi C, Caporossi T. Parasurgical therapy for keratoconus by riboflavin-ultraviolet type A rays induced cross-linking of corneal collagen. Preliminary refractive results in Italian study. J Cat Ref Surg 200632 837-845.

15. Spoerl E, Mrochen M, Sliney D, Troke S, Seiler T. Safety of UVA-riboflavin cross linking of the cornea. Cornea 2007 26 385-389.

16. Doors M, Tahzib NG, Eggink FA, Berendschot TTJM, Webers CAB, Nuijts RMMA. Use of anterior segment optical coherence tomography to study corneal changes after collagen cross-linking. Am J Ophthalmol 2009148 844-851.

17. Koller T, Iseli HP, Hafezi F, Vinciguerra P, Seiler T. Scheimpflug imaging of corneas after collagen cross-linking. Cornea 200928 510-515.

18. Mazzotta C, Balestrazzi A, Traversi C, Baiocchi S, Caporossi T, Tammasi C, Caporossi . Treatment of progressive keratoconus by riboflavin-UVA-induced cross-linking of corneal collagen. Ultrastructural analysis by Heidelberg retinal tomograph II in vivo confocal microscopy in humans. Cornea 200726 390-397. 
19. Grewal DS, Brar GS, Jain R, Sood V, Singla M, Grewal SPS. Corneal collagen crosslinking using riboflavin and ultraviolet-A light for keratoconus. One-year analysis using Scheimpflug imaging. J Cat Ref Surg 200935 425432.

20. Wollensak G, Spoerl E, Reber F, Seiler T. Keratocyte cytotoxicity of riboflavin/UVA treatment in vitro. Eye 2004 18 718-722.

21. Wollensak G, Spoerl E, Wilsch M, Seiler T. Keratocyte apoptosis after corneal collagen cross-linking using Riboflavin/UVA treatment. Cornea 200423 43-49.

22. Spoerl E, Huhle M, Seiler T. Induction of cross-links in corneal tissue. Exp Eye Res 199866 97-103.

23. Goldich Y, Barkana Y, Morad Y, Hartstein M, Avni I, Zadok D. Can we measure corneal biomechanical changes after collagen cross-linking in eyes with keratoconus? - A pilot study. Cornea 200928 498-502.

24. Kohlhaas M, Spoerl E, Schilde T, Unger G, Wittig C, Pillunat L. Biomechanical evidence of distribution of crosslinks in corneas treated with riboflavin and ultraviolet A light. J Cat Ref Surg 200632 279-283.

25. Mazzotta C, Balestrazzi A, Baiocchi S, Traversi C, Capriossi A. Stromal haze after combined Riboflavin-UVA corneal collagen cross- linking in keratoconus: in vivo confocal microscopy evaluation. Clin Exp Ophthalmol 200735 580-582.

26. Kymionis G, Portaliou D, Bouzoukis D, Suh LH, Pallikaris AI, Markomanolakis M, Yoo SH. Herpetic keratitis with iritis after corneal crosslinking with riboflavin and ultraviolet A for keratoconus. J Cat Ref Surg 200733 1982-1984.

27. Kymionis GD, Bouzoukis DI, Diakonis VF, Portaliou DM, Pallikaris AI, Yoo SH. Diffuse lamellar keratitis after corneal crosslinking in a patient with post-laser in situ keratomileusis corneal ectasia. J Cat Ref Surg 200733 2135-2137. 